\title{
CONTRIBUIÇÃO DA CIPESC® NA EXECUÇÃO DAS POLÍTICAS DE ATENÇÃO À SAÚDE DA CRIANÇA NO MUNICÍPIO DE CURITIBA, PARANÁ ${ }^{1}$ NURSING DIAGNOSES IN CHILDREN'S HEALTH CARE IN CURITIBA, BRAZIL: AN OVERVIEW OF CIPESC ${ }^{\circledR}$ BASES \\ DIAGNÓSTICOS DE ENFERMERÍA DE SALUD PÚBLICA INFANTIL EN CURITIBA, BRAZIL: CONTRIBUCIÓN DEL USO DE LA CIPESC®
}

\author{
Maira Rosa Apostolico², Márcia Regina Cubas ${ }^{3}$, Denise Meira Altino", Kelly Cristina Máxima Pereira \\ Emiko Yosbikawa Egry
}

${ }^{1}$ Trabalho integrante do projeto "As transformações nos processos de trabalho da enfermagem em Saúde Coletiva: instrumentalizando para uma prática inovadora”, coordenado pela Professora Doutora Emiko Yoshikawa Egry.

${ }^{2}$ Enfermeira pela Escola de Enfermagem da Universidade de São Paulo (USP). São Paulo, Brasil.

${ }^{3}$ Enfermeira. Mestre em Enfermagem. Doutora em Saúde Coletiva. Professora Assistente do Curso de Enfermagem da Pontifícia Universidade Católica do Paraná. Paraná, Brasil.

${ }_{5}^{4}$ Enfermeira pela Escola de Enfermagem da USP. São Paulo, Brasil.

${ }^{5}$ Enfermeira. Mestre em Enfermagem. Doutora em Saúde Pública. Livre Docente em Enfermagem em Saúde Coletiva. Professora Titular do Departamento de Enfermagem em Saúde Coletiva da Escola de Enfermagem da USP. Pesquisadora do Conselho Nacional de Desenvolvimento Científico e Tecnológico. Orientadora. São Paulo, Brasil.

PALAVRAS-CHAVE: Saúde da criança. Políticas de saúde. Diagnóstico de enfermagem. Saúde pública.
RESUMO: Trata-se de estudo descritivo e exploratório acerca do sistema de Classificação Internacional das Práticas de Enfermagem em Saúde Coletiva ${ }^{\circledR}$, adotado pela rede de atenção à saúde do munićpio de Curitiba, como parte de sua política de saúde. Foram coletados dados em fontes secundárias documentais e no banco de registro municipal. Os dados foram analisados à luz da Teoria de Intervenção Práxica da Enfermagem em Saúde Coletiva. Os resultados mostram que o município vem obtendo perfis epidemiológicos da saúde infantil melhores do que o restante do país e que a enfermagem tem contribuído para estes resultados, melhorando a qualidade de atenção, a partir do uso do sistema classificatório, que possibilita identificar as necessidades em saúde e os diagnósticos tanto os de desgaste quanto os de fortalecimentos.
KEYWORDS: Child health. Health policy. Nursing diagnosis. Public health.

\begin{abstract}
A classificatory system of nursing practice in collective health (International Classification of Nursing Practices in Collective Health ${ }^{\circledR}$ - Classificação Internacional das Práticas de Enfermagem em Saúde Coletiva ${ }^{\circledR}$ ) has been adopted in Curitiba's primary care health services as part of its policy. This study, descriptive and exploratory, seeks to show Nursing's contributions to epidemiological profile improvement in children's health. The data was collected through the reports about nursing diagnoses from the municipal computer database, and was analyzed using the Nursing Praxical Intervention Theory in Collective Health. The results have shown that children's health profiles in this municipality are better than in the remainder of the country. Nursing practices, qualified by using nursing diagnoses and a classificatory system, have allowed assessing improvement in health-illness processes. Finally nursing has been able to identify health needs and diagnoses, strengths and impairments of health-illness processes.
\end{abstract}

PALABRAS CLAVE: Salud del niño. Política de salud. Diagnóstico de enfermería. Salud pública.

RESUMEN: Se trata de un estudio descriptivo y exploratorio sobre la utilización del sistema de Clasificación Internacional de las Prácticas de Enfermería en Salud Colectiva ${ }^{\circledR}$ adoptado por la red de atención a la salud del municipio de Curitiba, como siendo parte de su política de salud. Los datos fueron recolectados a partir de fuentes secundarias documentales y del banco de registro municipal. Para el análisis de los datos fue utilizada la Teoría de Intervención Práxica de la Enfermería en Salud Colectiva. Los resultados obtenidos muestran que el municipio tiene perfiles epidemiológicos de salud infantil mejores que los del resto del país, y, que la enfermería ha contribuido para la obtención de esos resultados, mejorando la calidad de la atención por el uso del sistema de clasificación, lo que posibilita identificar las necesidades en salud y los diagnósticos, tanto relativos a los deterioros como a los fortalecimientos.

Endereço: Emiko Yoshikawa Egry

Av. Dr. Enéas de Carvalho Aguiar, 419

05.403-000 - Cerqueira César, São Paulo, SP, Brasil.

Email: maira_eeusp@yahoo.com.br ou emiyegry@usp.br
Artigo original: Pesquisa

Recebido em: 15 de fevereiro de 2007 Aprovação final em: 11 de julho de 2007 


\section{INTRODUÇÃO}

Os avanços na legislação brasileira desde a promulgação da Constituição Federal de 1988, garantiram direitos legais a grupos antes desconsiderados. Com a promulgação do Estatuto da Criança e do Adolescente, Lei 8.069 de 13 de julho de 1990 , a criança e o adolescente passam a gozar de igualdade quanto aos direitos fundamentais inerentes à pessoa humana, garantindo-lhes proteção, saúde, alimentação, educação, esporte, lazer, cultura, entre outros. Além disso, responsabiliza não só a família mas toda a sociedade e o poder público pelo cumprimento da lei. ${ }^{1}$

Estes avanços ocorreram globalmente, não somente no Brasil. A Organização Mundial da Saúde (OMS), Organização Panamericana de Saúde (OPAS), Fundo das Nações Unidas para a Infância (UNICEF), entre outros dedicam seu trabalho à saúde e bem estar de populações, incluindo crianças e adolescentes, com programas, protocolos e estudos sobre o assunto.

Em 1989, a Convenção sobre os Direitos da Criança adotada pela Assembléia Geral das Nações Unidas foi ratificada por 192 países, configurando o tratado mais endossado da história dos acordos mundiais. Essa convenção faz dos direitos da criança uma obrigação "moral e legal", obrigando os estados a assumirem a responsabilidade de cuidar de suas crianças. Além disso, os países firmaram em 2002, a Agenda do Milênio, com objetivos e metas definidas para os primeiros anos do século 21. Entre as medidas a serem alcançadas estão a erradicação de doenças e promoção da saúde; garantia de alimentação e educação; igualdade de direitos e diminuição dos índices de mortalidade infantil. Apesar disso, milhões de crianças ainda esperam medidas que melhorem suas condições de vida e têm nisso uma questão de sobrevivência. Trata-se de uma geração comprometida pelas dificuldades e necessidades; ainda que cheguem à idade adulta, terão sua saúde e seu futuro drasticamente comprometidos por falta de perspectivas e possibilidades de crescimento social, político e econômico. Há uma parcela de crianças no mundo que está à margem da sociedade e além de excluídas do acesso aos serviços essenciais, estará totalmente excluída dos processos políticos de seus países, em virtude de guerras, conflitos e políticas injustas e discriminatórias. ${ }^{2}$

Dentro desse panorama, Curitiba, capital do Estado do Paraná, destaca-se pelas políticas de saúde planejadas para atender as necessidades de saúde e desenvolvimento das crianças que, nos últimos anos, tem obtido redução dos mais importantes indicadores do município. Conta com a organização política e envolvimento de diferentes profissionais, trabalhando em frentes como saúde, educação, assistência social, cultura, lazer e desenvolvimento. ${ }^{3}$

Envolvidos nesse processo, estão os profissionais de saúde do município, desempenhando importante papel na realização dos programas e alcance das metas propostas. A rede de atenção básica constituída pelas unidades de saúde do município constitui a porta de entrada do Sistema de Saúde, atuando em ações de promoção e prevenção de agravos, através dos diversos programas instituídos pela Prefeitura Municipal de Curitiba. ${ }^{3}$ A Enfermagem aparece em destaque, com intensa atuação nesse processo, desde a identificação das necessidades da população até o efetivo atendimento, como por exemplo através da consulta de enfermagem realizada no âmbito da atenção primária. ${ }^{4}$

Para isso, dispõe de importante ferramenta a Classificação Internacional das Práticas de Enfermagem em Saúde Coletiva (CIPESC"), integrada ao prontuário eletrônico. Em 2001, após a Associação Brasileira de Enfermagem apresentar os resultados do projeto CIPESC1 na Conferência Municipal de Curitiba, um grupo de enfermeiras, de posse desses achados e com intuito de renovar e reestruturar a enfermagem voltada para a atenção básica do município, constituiu o grupo condutor da Sistematização das Práticas de Enfermagem em Saúde Coletiva da SMS, buscando meios e referências para a construção de uma sistematização da assistência de enfermagem que atendesse às necessidades do município, no contexto do Sistema Único de Saúde (SUS) e com o devido enfoque na saúde coletiva. ${ }^{4}$

O desafio era imenso, partindo desde a escolha da teoria de enfermagem que melhor definiria o trabalho até mesmo a operacionalização das tarefas, visto que o grupo acumulava também seus encargos de origem, na academia ou mesmo no serviço público do município..$^{4.5}$

A escolha da Teoria das Necessidades Humanas Básicas de Wanda de Aguiar Horta respeitou entre outros, um aspecto fundamental desse trabalho: o fato da maior parte dos profissionais enfermeiros da rede, vindos das escolas do Paraná, terem em sua formação acadêmica, contato com tal teoria e, para muitos, incorporada no seu próprio modo de cuidar. ${ }^{5}$ Essa teoria está sob referencial humanista, admitindo que as experiências vividas pelo indivíduo diferem segundo o contexto social e familiar em que aparecem. ${ }^{6}$ 
O foco no indivíduo e em suas necessidades básicas permite identificar também necessidades da família ou de outros grupos sociais, abrindo-se uma grande fresta para seu uso nas questões coletivas, sobretudo aquelas relacionadas à vulnerabilidade.

Além disso, a aproximação do trabalho à esfera coletiva foi feita através da Teoria da Intervenção Práxica de Enfermagem em Saúde Coletiva (TIPESC), ${ }^{5}$ que traz em seus momentos a captação da Realidade Objetiva (RO), a interpretação da RO, a construção do Projeto de Intervenção na $\mathrm{RO}$, a intervenção propriamente dita na $\mathrm{RO}$ e a reinterpretação da RO. Esse mesmo referencial foi utilizado no projeto CIPESC1. A aproximação das enfermeiras à teoria aconteceu a partir de estudos do grupo e oficinas de trabalho.

Na primeira etapa, foi construída a ferramenta para atender consultas da área temática saúde da mulher, implantada em julho de 2004. Em junho de 2005 passou a atender também a área temática Saúde da Criança, com diagnósticos e intervenções de enfermagem que são utilizadas nas consultas de enfermagem para esse público, permitindo viabilizar as ações e as necessidades das crianças de Curitiba. ${ }^{5}$

É nesse panorama que a Saúde da Criança será observada e descrita nesse trabalho, ou seja, através das políticas de saúde do município, incluindo programas de atenção à criança que direcionam cuidados desde a gestação até a adolescência e idade adulta, bem como os recursos disponibilizados pela Secretaria Municipal de Saúde para monitorizar e acessar esse grupo de usuários do serviço.

Este estudo integra o projeto "As transformações nos processos de trabalho da enfermagem em Saúde Coletiva: instrumentalizando para uma prática inovadora", ocupando-se de parte do objetivo específico de "captar a realidade objetiva da implantação da Sistematização da Assistência de Enfermagem na rede de Unidades de Saúde de Curitiba, buscando as contradições entre as dimensões: estrutural, particular e singular" .5:121

Tem por objetivo geral descrever os diagnósticos e intervenções de enfermagem gerados nas consultas de enfermagem na atenção à criança, tendo por base o sistema CIPESC $\square$ e para isso teve por objetivos específicos: a) descrever o perfil morbimortallidade das crianças de Curitiba e os principais programas municipais dirigidos à atenção básica saúde da criança; b) identificar os diagnósticos que são potentes para refletir a face coletiva das crianças atendidas na rede municipal de atenção básica de Curitiba e c) correlacionar os diagnósticos e intervenções de enfermagem gerados nas consultas com o perfil morbi-mortalidade das crianças de Curitiba.

\section{METODOLOGIA}

A base metodológica utilizada foi a Teoria de Intervenção Práxica de Enfermagem em Saúde Coletiva (TIPESC): “[...] na sua vertente metodológica, é a sistematização dinâmica de captar e interpretar um fenômeno articulado aos processos de produção e reprodução social referentes à saúde e a doença de uma dada coletividade, no marco de sua conjuntura e estrutura, dentro de um contexto social historicamente determinado [...]" .:103

Neste trabalho, foi abordado o primeiro e segundo momentos da TIPESC, que compreende a Captação da $\mathrm{RO}$ e Interpretação da RO, servindo de subsídio para os demais momentos em oportunidade vindoura.

Os preceitos éticos foram resguardados pela apreciação do Projeto Unificado "As Transformações dos Processos de Trabalho da Enfermagem em Saúde Coletiva: instrumentalizando para uma prática inovadora", ${ }^{8}$ do qual este trabalho é parte, pelo Comitê de Ética da Escola de Enfermagem da Universidade de São Paulo, sob Processo No 415/2004/CEP-EEUSP, e pelo Comitê de Ética em Pesquisa da Secretaria Municipal de Saúde de Curitiba, Protocolo $\mathrm{N}^{\mathrm{o}}$ 102/2004, dando cumprimento à resolução 196/96 do Conselho Nacional de Saúde. 5

Sendo parte de um projeto maior, este trabalho pôde lançar mão de dados anteriormente coletados. A descrição do perfil morbi-mortalidade do município partiu de dados disponibilizados pela Secretaria Municipal de Saúde (SMS) de Curitiba em visita realizada no mês de dezembro de 2005 , bem como o material referente aos diagnósticos de enfermagem. Além disso, o conteúdo dos sites oficiais de Curitiba contribuiu com o trabalho, visto que os sites da capital do Paraná são completos e atualizados com freqüência, facilitando o trabalho das pesquisadoras. Foram visitados os bancos de dados e sites oficiais como: Instituto de Pesquisa e Planejamento Urbano de Curitiba (IPUC), Instituto Brasileiro de Geografia e Estatística (IBGE), OMS, OPAS, UNICEF, e outros. A análise dos dados foi feita à luz de bibliografia pertinente, após busca bibliográfica em bases de dados como Literatura Latino-Americana e do Caribe em Ciências da 
Saúde (LILACS), Medical Literature Analysis and Retrieval System Online (MEDLINE), Scientific Electronic Library Online (SciELO), entre outras. Os dados quantitativos foram trabalhados em base Excel ${ }^{\circledR}$, utilizando-se de estatística simples.

\section{RESULTADOS E DISCUSSÃO}

Curitiba, capital do Estado do Paraná, conta com uma área de $430,9 \mathrm{Km} 2$ e uma população de 1.727.010 habitantes, segundo estimativa do IBGE para o ano de 2004. O município é dividido em 75 bairros, organizados em 9 Administrações Regionais: Bairro Novo, Boa Vista, Boqueirão, Cajurú, CIC, Matriz, Pinheirinho, Portão e Santa Felicidade. Está inserido em uma região metropolitana com 26 municípios que experimentou uma taxa de crescimento de $3,4 \%$ de 2000 a 2006. O Produto Interno Bruto (PIB) de Curitiba em 2003 foi o maior de sua história, chegando a $\mathrm{R} \$ 32,7$ bilhões.

A pirâmide populacional apresenta base larga tendendo a estreitar-se, característica de populações que estão a caminho do envelhecimento. A esperança de vida ao nascer em 1980 era de 65,53 anos. Em 2004, a estimativa era de 72,90 anos para mulheres e 68,69 anos para os homens. Em relação ao analfabetismo, para jovens e adultos com mais de 15 anos de idade, Curitiba apresenta o menor índice $(3,36)$ quando comparada a outras capitais brasileiras como Florianópolis $(3,56)$, Porto Alegre $(3,45)$, São Paulo $(4,89)$ e Recife $(10,55){ }^{9}$

O setor de serviços é o predominante em relação aos empregos oferecidos em Curitiba, representando no ano 2000 quase $66 \%$ da atividade econômica do município, seguido da Indústria (18\%) e Comércio (15\%). O PIB de Curitiba em 2003 superou o índice do país, calculado em R $\$ 9.105,15$ contra $\mathrm{R} \$ 8.694,47$ o valor nacional.

O número de domicílios de Curitiba chega a 471.163, segundo o senso de 2000 realizado pelo IBGE e são chefiados em sua maioria por homens. Do total de 470.014 (100\%) domicílios totalizados pelo IBGE, $334.321(71,13 \%)$ domicílios são chefiados por homens. Destes, mais de $28 \%$ dos chefes de família tem de 11 a 14 anos de instrução, seguidos por mais de $25 \%$ com 4 a 7 anos de instrução e $18,13 \%$ com 15 anos ou mais. Aproximadamente $12 \%$ tem até 3 anos de instrução. Para as mulheres, a situação é semelhante, de que cerca de $19 \%$ dos domicílios chefiados por mulheres, estas tem até 3 anos de instrução. ${ }^{9}$
Quanto ao rendimento dos responsáveis por domicílios, também segundo o Censo de 2000 do IBGE, 23,34\% dos homens recebem mais de 5 a 10 salários mínimos mensais, enquanto $19,33 \%$ das mulheres enquadram-se nessa faixa de salário. Para rendimento mensal acima de 20 salários mínimos, aparecem $11,08 \%$ dos homens e apenas $5,07 \%$ das mulheres responsáveis por domicílios de Curitiba, apontando a desigualdade de renda e menor ganho salarial pelas mulheres. ${ }^{9}$

\section{Política de atenção à criança}

O processo de municipalização do sistema de saúde teve início em 1992, e implantou o Programa Saúde da Família em 1996, embora operacionalizasse a estratégia desde 1991, já operando em um sistema descentralizado em regionais administrativas. Aderiu à Gestão Plena dos Serviços Municipais em 1998, o que permitiu à SMS ampliar investimentos e privilegiar a atenção aos problemas de maior peso epidemiológico. ${ }^{3}$

O SUS de Curitiba é integrado por 281 prestadores ambulatoriais (135 públicos, 17 filantrópicos e os demais privados), a assistência hospitalar é exercida por 30 hospitais credenciados (três públicos, 12 filantrópicos e 15 privados), totalizando 4.735 leitos. O serviço municipal dispõe de uma rede própria com nove Distritos Sanitários agregando um hospital geral e maternidade com 60 leitos, um laboratório municipal e 110 Unidades de Saúde com 133 equipes de Saúde da Família e 1.164 Agentes Comunitários de Saúde. Existe um investimento sistemático por parte da SMS em equipamentos e tecnologia de informática, como com o prontuário eletrônico (médico, enfermagem e odontologia, além de outros aplicativos). ${ }^{3}$

As principais causas de internação pelo SUS, segundo a SMS em 2005 são: gravidez, parto e puerpério (18,49\%), doenças do aparelho circulatório $(10,00 \%)$, neoplasias $(8,64 \%)$, doenças do aparelho respiratório $(8,18 \%)$, doenças do aparelho digestivo $(7,83 \%)$, doenças do aparelho geniturinário $(4,92 \%)$ e outras causas que incluem causas externas (41,94\%). Esse panorama não difere consideravelmente do quadro nacional. ${ }^{9}$

As ações de saúde desenvolvidas pela SMS são divididas em áreas temáticas, que englobam tanto programas oficiais preconizados pelo Ministério da Saúde como outros programas criados por iniciativa da própria SMS.

Dentre os inúmeros programas desenvolvidos pela SMS, existem aqueles exclusivos à Saúde da 
Criança e outros que garantem a melhoria do atendimento e da saúde da população em geral. A SMS vem trabalhando na construção do "Protocolo de Atenção à Saúde Integral da Criança”, visando normalizar o atendimento de crianças de $\mathrm{O}$ a 10 anos de idade, construindo cronogramas de consultas, procedimentos realizados nas Unidades de Saúde, cuidados com o bebê e criança em todos os aspectos (amamentação, nutrição, crescimento e prevenção de doenças e acidentes).

Os principais programas desenvolvidos pela SMS, que buscam atender as necessidades das crianças, estão descritos a seguir.

Programa Mãe Curitibana: desde março de 1999, tem como objetivo primeiro a melhoria na qualidade de pré-natal em todos os aspectos, aumentando a qualidade e segurança do atendimento às gestantes e às crianças de Curitiba. Garante que a gestante realize todos os exames de pré-natal e conheça a maternidade onde será realizado o parto. Resultou na ordenação do fluxo de referência e contra-referência entre os serviços existentes no município, qualificação dos profissionais, organização e melhoria no acesso das gestantes ao prénatal e assistência ao neonato com ampliação de leitos para recém-nascidos de médio e alto risco. $\mathrm{O}$ programa Mãe Curitibana é considerado o maior responsável pela queda dos índices de mortalidade infantil e redução expressiva dos casos de AIDS em crianças, devido à implantação das medidas de prevenção da transmissão vertical. ${ }^{9}$

Programa Nascer em Curitiba: trata-se do monitoramento das crianças com risco ao nascer $\mathrm{e}$ risco evolutivo, direcionando as ações como atendimento priorizado nas Unidades de Saúde e Visitas Domiciliárias que permitem ao profissional de saúde orientar a família a respeito dos cuidados, vacinas e aleitamento materno. ${ }^{3}$

Programa de Combate às Carências Nutricionais (SISVAN): vigilância nutricional através de um sistema de coleta, processamento e análise contínuos de dados da população, permitindo caracterizar grupos de risco e subsidiando a formulação de políticas, programas e intervenções. Funciona nas Unidades Municipais de Saúde, Escolas da Rede Pública de Ensino e Maternidade de Curitiba. Tem como alvo os recém nascidos, crianças menores de 5 anos, escolares, gestantes, adultos e idosos. Nas unidades de saúde os dados antropométricos são coletados nas consultas de enfermagem e lançados diretamente no prontuário eletrônico. Periodicamente são feitas campanhas juntamente com campanhas de vacinação para coleta de dados antropométricos e de aleitamento materno de crianças menores de 5 anos, obtendo-se um perfil nutricional do município. Além disso, também periodicamente, são feitas dosagens amostrais de hemoglobina, verificando a prevalência de anemia na população menor de 5 anos de idade. Nas escolas, a participação é feita através de dados coletados anualmente por professores de Educação Física, tais como dados antropométricos, biopedância e hábitos alimentares. ${ }^{3}$

Programa de Suplementação Nutricional: implantado em 2003, objetiva combater a desnutrição em crianças menores de 2 anos de idade, com a distribuição mensal de fórmula e leite enriquecido para 1.100 crianças usuárias de Rede Municipal de Saúde. Funciona concomitantemente com o programa Bolsa Alimentação, implantado pelo Governo Federal, que distribui quantias em dinheiro para famílias carentes. O critério de inclusão das famílias beneficiadas é diferente, por isso a SMS decidiu manter o programa original. As crianças são acompanhadas mensalmente nas unidades de saúde e participam de oficinas educativas juntamente com seus familiares. O tempo de permanência no programa é de no mínimo 1 ano. O programa tem tido avaliação altamente positiva por parte dos usuários, entretanto, em 2005 , persistia ainda, um grupo de crianças (14\%) que não apresentou crescimento satisfatório, demonstrando a determinação social da desnutrição e apontando para outras dimensões da vida que devem ser identificadas e trabalhadas no processo saúde-doença. ${ }^{3}$

Rede de Proteção à Criança e ao Adolescente em Situação de Risco no município de Curitiba: estruturada com a participação de entidades dos diversos setores que atendem crianças, essa iniciativa tem por objetivo reduzir o problema da violência infra-familiar, intervir em situações geradoras de conflitos e maus tratos, oferecer atendimento às famílias, tanto para agressores quanto para agredidos e sobretudo, conhecer o problema e construir maneiras de enfrentamento. A "Rede" construiu o trabalho sob nova concepção, permitindo a todo tempo, a inclusão de novos parceiros e novas iniciativas governamentais ou não governamentais. Não implicou em investimentos significativos, mas em uma mudança de paradigma: o olhar dos profissionais precisou ser mudado e a percepção para diagnosticar precocemente situações de risco, mais acurada. A integração dos programas e recursos existentes foi a chave da mudança. A partir de uma situação de violência identificada por qualquer pro- 
fissional (professor, educador, médico, pedagogo, enfermeira, psicólogo, dentista e outros), desencadeia-se um processo de busca de informações que permitam avaliar a situação de risco e classificá-lo em leve, moderado ou grave. Para os casos leves, é preenchido um protocolo de acompanhamento e encaminhado para as instituições em que a criança mantém contato, além da orientação da família. Nos casos mais graves, além do protocolo, são comunicados os órgãos oficiais como Conselho Tutelar, SOS Criança e encaminhada a vítima a um serviço de saúde. A liberação da criança só ocorre após garantida a sua proteção pelo Conselho Tutelar. Existe uma preocupação especial em relação ao sigilo das informações. $\mathrm{O}$ acompanhamento de cada integrante da rede ocorre conforme sua competência: à saúde cabe diagnosticar e encaminhar casos aos serviços de saúde mental, à educação cabe o acompanhamento pedagógico da vítima na tentativa de minimizar dados decorrentes da violência e integração dos pais/família em grupos de orientação e acompanhamento; ao serviço social cabe o acompanhamento nos casos em que as condições precárias de vida das famílias são os potencializadores da violência doméstica. Em 2004 foram realizados 1986 atendimentos sendo que $92,3 \%$ relativos às violências doméstica e no primeiro semestre de 2005, 995 notificações contra crianças e adolescentes de Curitiba sendo $85,2 \%$ referentes à violência doméstica. ${ }^{3}$

Pacto pela Vida: trata-se do esforço para a redução da mortalidade infantil através de um conjunto de ações compartilhadas entre a SMS e governo do Estado. ${ }^{3}$

Acolhimento Solidário: refere-se à reorganização do processo de trabalho, transformando a prática dos profissionais de saúde. Tem como objetivo abrir os serviços de saúde para a deman$\mathrm{da}$, humanizando o atendimento e buscando a satisfação do usuário. Foi necessário capacitar os profissionais e elaborar manuais e protocolos, com a participação efetiva das equipes e pactuação com os Conselhos Locais de Saúde. Os atendimentos são realizados buscando a priorização dos casos mais graves, através de avaliação da enfermagem, amparada pelos protocolos de atendimento, garantindo a resolubilidade no atendimento. ${ }^{3}$

\section{Panorama da saúde da criança}

O panorama da saúde da criança em Curitiba aponta para a queda de indicadores importantes nos últimos tempos. Em 1996 foi a introduzida da vacina anti HIB (Hemófilos influenza $B$ causador de Meningite) na vacinação de rotina das crianças, resultando em queda considerável nos casos: passaram de 35 casos por 100 mil crianças em 1996 para menos de 1 caso por 100 mil crianças em 2004. Para o sarampo, Curitiba seguiu a tendência Nacional, embora tenha conseguido interromper a doença em 1998, dois anos antes do último caso de Sarampo do Brasil. Em 1997 e 1998, o surto de sarampo iniciado em São Paulo e que se espalhou para outras localidades do país, motivou uma grande campanha de vacinação que atendeu mais de 600 mil pessoas de um a 39 anos de idade na capital paranaense. Combinada com a cobertura vacinal de rotina e campanhas nacionais de seguimento, o sucesso na interrupção da doença foi alcançado antes e permitiu que Curitiba superasse os $95 \%$ de cobertura vacinal das crianças de até 4 anos de idade, com nenhum caso registrado desde 1998. Importante ressaltar que ainda existem casos de sarampo registrados na América Latina, e demais continentes o que torna necessária a vigilância epidemiológica intensa para manter interrompidos os casos no Brasil. ${ }^{3}$

A análise do perfil de nascidos vivos do município no período de 2000 a 2004, revela uma queda no número de nascimentos de mães residentes em Curitiba. Houve queda também no número de gestações de adolescentes e um aumento das gestantes com 30 anos ou mais de idade. Aumentou o número de mães com 8 ou mais anos de escolaridade e o número de cesáreas também cresceu, com conseqüente diminuição dos partos normais. Em média, $77,6 \%$ das gestantes do período realizaram 6 ou mais consultas de pré-natal. Segundo relatório anual da UNICEF, divulgado em dezembro de 2006, a escolaridade das mães reflete diretamente nos índices de mortalidade infantil. ${ }^{2}$ Somado a isso, o programa de atenção à gestante e às crianças de Curitiba contribuem para esse quadro. Entretanto, o número crescente de gestações em mulheres com mais de 30 anos, também altera os números de partos cesárea, visto que aumenta a possibilidade de distócias.

De 1979 a 2004 o coeficiente de mortalidade infantil sofreu uma redução de $76,4 \%$, com queda mais acentuada a partir do final da década de 90 . Curitiba se mantém sempre abaixo do coeficiente nacional e estadual, chegando à marca de 11,3 óbitos por 1000 nascidos vivos para menores de 1 ano.'

No ano de 2005, Curitiba apresentou aumento nos coeficientes de mortalidade por afecções 
neonatais ligados a prematuridade e baixo peso ao nascer. Uma das causas identificadas pelos serviços de saúde foi o número aumentado de ocorrências de infecções do trato urinário nas gestantes. Identificada essa possível causa, os serviços de saúde foram orientados através de protocolos de atenção à gestante nessas situações, com a preocupação de reverter o aumento do indicador?

Essas taxas traduzem de modo sensível a qualidade de saúde da população, principalmente referente às condições de moradia, alimentação, educação, saneamento e acesso aos serviços de saúde.

\section{A CIPESC ${ }^{\circledR}$ e a saúde da criança}

Para compreender a realidade assistencial de enfermagem de Curitiba, o primeiro momento do Projeto Unificado buscou identificar as práticas de enfermagem executadas pelos profissionais da rede municipal de Curitiba, através de um check-list. ${ }^{5} \mathrm{O}$ resultado desse check-list mostrou que $100 \%$ dos enfermeiros entrevistados realizam a consulta de enfermagem diariamente, além de outras atividades diárias e semanais como a visita domiciliária, orientação aos grupos de usuários, coordenação e supervisão dos profissionais da enfermagem na Unidade de Saúde, entre outras atividades. ${ }^{9-13}$

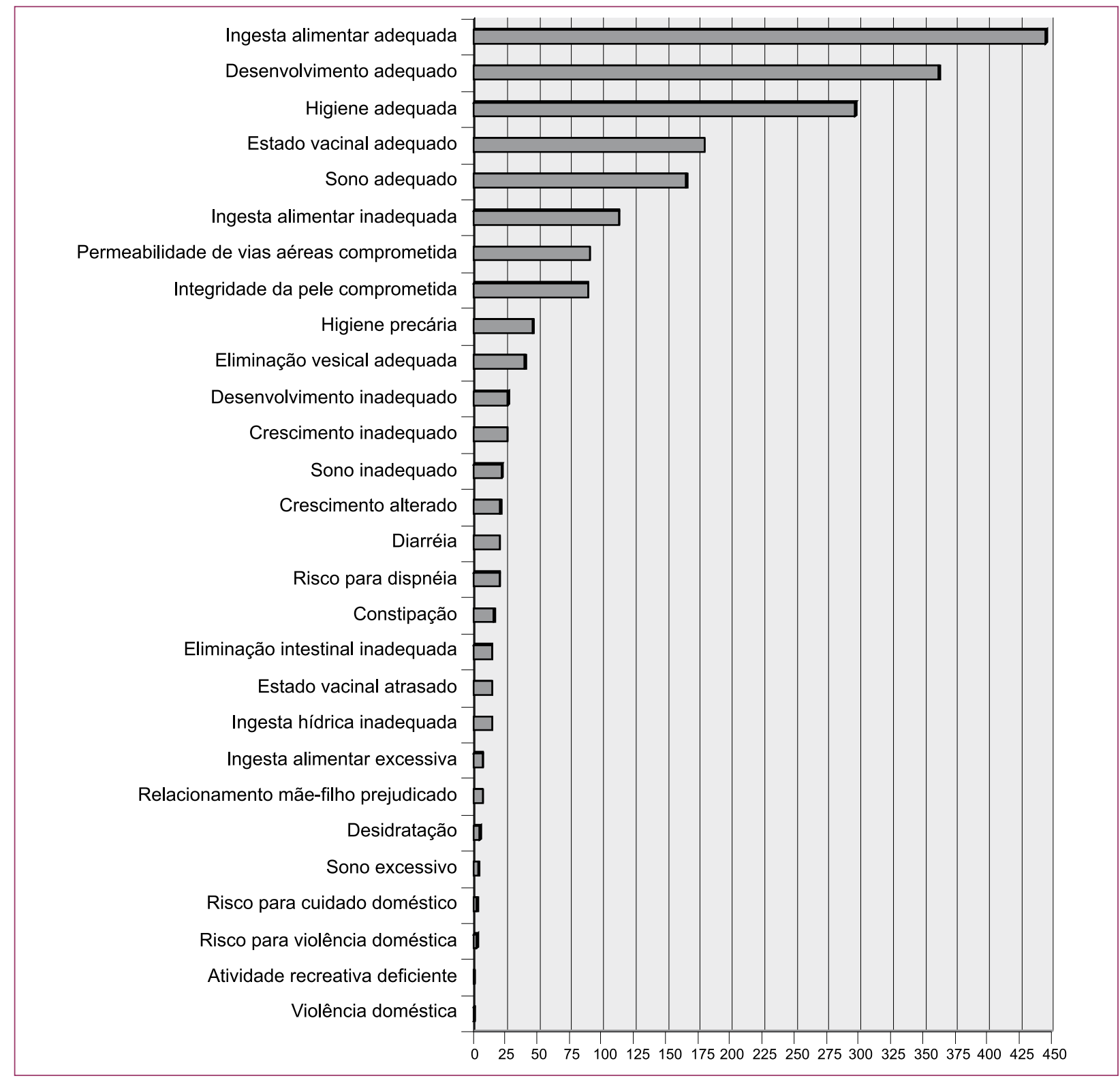

Figura 1 - Diagnósticos de enfermagem gerados nas consultas de enfermagem no período de agosto a outubro de 2005, Curitiba. 
Foram realizadas, no ano de 2005 , um total de 239.412 consultas de enfermagem na rede municipal de saúde, conforme dados disponibilizados pela SMS. Em uma parte dessas consultas que foram direcionadas às crianças a utilização da ferramenta CIPESC ${ }^{\circledR}$ gerou diagnósticos e Intervenções de enfermagem que podem ser vistos em relatórios consolidados, que mostram a produção dos enfermeiros nas consultas realizadas.

No período de Agosto a Outubro de 2005 foram gerados 28 diagnósticos nas consultas realizadas na área temática Saúde da Criança distribuídos na seguinte freqüência.

Observa-se que os diagnósticos que mais aparecem nesse período estão de acordo com os principais programas desenvolvidos pela SMS, como por exemplo, os diagnósticos referentes à alimentação, crescimento e desenvolvimento e estado vacinal das crianças.

Através dos diagnósticos é possível perceber que as consultas de enfermagem em saúde da criança trazem um conteúdo de puericultura bastante grande, com destaque para os diagnósticos de fortalecimento do indivíduo frente aos processos de saúde-doença (ingesta alimentar, crescimento, desenvolvimento e estado vacinal adequados), que aparecem em maior número do que os diagnósticos de desgaste do indivíduo frente aos processos de saúde-doença.

Em relação aos diagnósticos relacionados ao tema segurança, embora apareçam em uma freqüência muito pequena, estiveram presentes nas consultas do período analisado. De acordo com o programa Rede de Proteção à Criança e ao Adolescente em Situação de Risco no município de Curitiba, no primeiro semestre de 2005 foram feitas 995 notificações. Nos meses de junho e julho do mesmo ano, a CIPESC ${ }^{\circledR}$ captou apenas 1 caso de violência contra a criança, e outros poucos casos de situações de risco. A violência doméstica pode ser também avaliada no sentido cultural. A violência, em muitos casos, apóia-se na crença de que a criança é propriedade da família, tentando justificar agressões físicas e psicológicas, silenciosas para grande parte da sociedade. A estruturação do sistema de saúde vem favorecendo a comunicação e registros de casos de violência e aumentando a resposta social nesses casos, sobretudo contra a criança. ${ }^{10}$ É importante ressaltar que estando o diagnóstico presente este constitui importante ferramenta de auxílio ao programa de proteção à criança já implantado na rede municipal de Curiti- ba, captando desde situações como relacionamento prejudicado até o risco para a violência.

Os diagnósticos são indicadores de necessidades de cuidados de enfermagem. São favoráveis ao desenvolvimento do corpo de conhecimento da enfermagem na medida em que geram intervenções e necessidade de estudo a respeito delas. Por sua vez, através das intervenções é possível avaliar o processo de tomada de decisão e fundamentar a avaliação dos resultados. ${ }^{11}$

A CIPESC ${ }^{\circledR}$ é usada principalmente como ferramenta do processo assistencial. Além disso, é um importante instrumento de avaliação do processo de trabalho, favorecendo a organização e as atividades de gerenciamento. Pela sua forma flexível, permite que a Classificação se ajuste à realidade local e não o contrário. Portanto, “a CIPESC ${ }^{\circledR}$ é um potente instrumento de trabalho do enfermeiro em saúde coletiva, tanto do ponto de vista assistencial, quanto de gerência e de investigação". ${ }^{12: 32}$

$\mathrm{Na}$ consulta de enfermagem que utiliza a base CIPESC ${ }^{\circledR}$, no mínimo um e no máximo quatro diagnósticos devem ser escolhidos; para cada diagnóstico é obrigatória a escolha de uma a quinze intervenções de enfermagem. ${ }^{5}$ Através de relatórios disponibilizados pela SMS de Curitiba foram identificadas as intervenções de enfermagem geradas nos meses de junho e julho de 2005. No total foram 9.566 intervenções geradas no período, distribuídas em 194 intervenções diferentes. Deste total, 5.969 (62\%) delas são de responsabilidade do enfermeiro executar enquanto as 3.597 (38\%) restantes são de responsabilidade do usuário. Isso mostra o envolvimento dos profissionais da saúde com o cuidado e atenção à necessidade do indivíduo atendido.

O inventário vocabular de fenômenos e ações de enfermagem em saúde coletiva aponta para a importância de se diferenciar ação de enfermagem e intervenção de enfermagem. ${ }^{14}$ De acordo com o CIE, a ação de enfermagem se refere ao desempenho do enfermeiro na prática, enquanto a intervenção de enfermagem é uma ação em resposta a um diagnóstico de enfermagem. Com base nesse critério e no inventário de ações descrito pelas autoras como resultado do projeto CIPESC1, as intervenções de enfermagem de responsabilidade do profissional puderam se divididas em quatro grupos a partir de um conceito central: atender, gerenciar, informar e observar. Cada um desses grupos, por sua vez, abrangem outros verbos que agrupam as ações propriamente ditas. 
As intervenções que mais foram escolhidas nas consultas de enfermagem são representadas pelo grande verbo Informar, sendo as intervenções de orientação as mais freqüentes no período analisado, seguidas de ensinar e explicar. O segundo grande verbo mais presente foi observar, sendo a ação mais freqüente enquadradas no verbo Pesquisar, que engloba anamnese e vigilância epidemiológica, entre outras.

\section{CONSIDERAÇÕES FINAIS}

Muitos avanços são observados desde a promulgação da Constituição Brasileira de 1988, ditando novas diretrizes para a saúde do brasileiro. ${ }^{15}$ Tornou-se um desafio importante para os municípios, reestruturar sua atenção básica e promover a saúde de sua população com resolubilidade. Curitiba, desde então, vem trabalhando no sentido de consolidar os princípios do SUS. Isso se reflete nos indicadores que ao longo dos anos vêm apresentando mudanças significativas. Certamente o investimento da Prefeitura Municipal de Curitiba não é direcionado exclusivamente à saúde. Também existem investimentos importantes em infra-estrutura urbana, educação de crianças, jovens e adultos, ações de vigilância sanitária e epidemiológica, reestruturação física e administrativa de serviços de saúde favorecendo a resolubilidade dos problemas da população, entre outros. ${ }^{3}$

As questões de saúde da população curitibana, mesmo com a participação social através das conferências locais, distritais e municipais, ainda são tratadas em uma lógica voltada para os aspectos biológicos do indivíduo. A Enfermagem tem condições de contribuir fortemente com seus fenômenos, pois é sensível para capturar as necessidades do indivíduo à luz da determinação social, ou seja, como sujeito partícipe e referente a um dado coletivo. ${ }^{12}$ O trabalho multidisciplinar é imprescindível, entretanto, para a enfermagem CIPESC ${ }^{\circledR}$ tem sido uma ferramenta fundamental, para qualificar o trabalho e contribuir de forma definitiva nos processos de intervenção da saúde/doença das populações. Mais ainda, ela deve ser utilizada com análise e reflexão, ou seja, sem nunca deixar a crítica, pois de outro modo "a enfermagem corre o risco de fazer uma avaliação simplista de sua atuação", deixando de utilizar sua percepção para alterar cenário onde atua. ${ }^{16}$

Esse é o panorama da saúde da criança de Curitiba. As políticas de saúde desenvolvidas pelo município visam de fato a melhoria não só do atendimento à criança doente, mas, principalmente, a melhoria da qualidade de vida da população em geral. A enfermagem atua como peça fundamental nesse processo. Seu saber e sua prática são essenciais para reconhecer as necessidades da população e propor intervenções efetivas. Além disso, a CIPESC ${ }^{\circledR}$ se configura como um instrumento valioso no processo de trabalho da enfermagem em saúde coletiva, bem como possibilita capturar as necessidades de saúde das crianças curitibanas, contribuindo definitivamente à consecução das metas definidas nos planos diretores do Município.

\section{REFERÊNCIAS}

1 Brasil. Lei No 8.069, de 13 de Julho de 1990. Dispõe sobre o Estatuto da Criança e do Adolescente e dá outras providências [acesso em 2006 Out 02] Disponível em: www.planalto.gov.br/ccivil/LEIS/ L8069.htm

2 Fundo das Nações Unidas para a Infância [página de Internet]. Brasília (DF): UNICEF; [acesso em 2006 Out 02]. Disponível em: http://www.unicef.org/brazil

3 Secretaria Municipal de Saúde de Curitiba [página de Internet]. Curitiba (PR): SMS; [acesso em 2006 Out 04]. Disponível em: http://www.curitiba.pr.gov. $\mathrm{br} / \mathrm{saude} / \mathrm{sms} /$ index.htm

4 Albuquerque LM, Cubas MR, organizadoras. Cipescando em Curitiba: construção e implementação da nomenclatura de diagnósticos e intervenções de enfermagem na rede básica de saúde. Curitiba (PR): ABEn-PR; 2005.

5 Egry EY, Cubas MR, organizadoras. O trabalho da enfermagem em saúde coletiva no cenário CIPESC $^{\circledR}$ guia para pesquisadores. Curitiba (PR): ABEn-PR; 2006. p.121.

6 Gualda DMR. Fundamentação teórico-conceitual do processo de cuidar. In: Cianciarullo TI, Gualda DMR, Melleiro MM, Anabuki MH, organizadores. Sistema de assistência de enfermagem: evolução e tendências. São Paulo (SP): Ícone; 2001.

7 Egry EY. Saúde Coletiva construindo um novo método em Enfermagem. São Paulo (SP): Ícone; 1996.

8 Egry EY, Cubas MR, organizadoras. O trabalho da enfermagem em saúde coletiva no cenário CIPESC guia para pesquisadores. Curitiba (PR): ABEn-PR; 2006. p.117-43.

9 Prefeitura Municipal de Curitiba, Instituto de Pesquisa e Planejamento Urbano de Curitiba, Setor de Monitoração. Banco de Dados [página de Internet]. Curitiba (PR): PMC/IPPUV; 2006 [acesso em 2006 Out 02]. Disponível em: http://www.curitiba.gov.br 
10 Ricas J, Donoso MTV, Gresta MLM. A violência na infância como uma questão cultural. Texto Contexto Enferm. 2006 Jan-Mar; 15 (1): 151-4.

11 Cruz DALM. Constribuições do diagnóstico de enfermagem para a autonomia da enfermeira. In: Guedes MVC, Araújo TL, organizadoras. O uso do diagnóstico na prática da enfermagem. Brasília (DF): ABEn; 1997. p.51-8.

12 Cubas MR. CIPESC ${ }^{\circledR}$ Curitiba: proposta de uma ferramenta re-leitora da face coletiva do processo saúde-doença [tese]. Doutorado. São Paulo (SP): USP/ Programa de Pós Graduação em Enfermagem; 2006.

13 Altino DM, Duarte FO, Apostólico MR. Classificação das Práticas de Enfermagem em Saúde Coletiva - CIPESC ${ }^{\circledR}$ : captação da realidade objetiva cenário
Curitiba-PR [projeto de pesquisa]. São Paulo (SP): USP/Escola de Enfermagem; 2005.

14 Garcia TR, Nóbrega MML. Projeto CIPESC ${ }^{\circledR}$ - CIE/ABEn: inventário vocabular de fenômenos e ações de enfermagem em saúde coletiva. In: Garcia TR, Nóbrega MML, organizadoras. Sistema de Classificação da Prática de Enfermagem: um trabalho coletivo. João Pessoa (PB): ABEn; 2000.

15 Presidência da República (BR), Casa Civil. Constituição da República Federativa do Brasil de 1988 [acesso em 2006 Out 02]. Disponível em: http://www.planalto. gov.br/ccivil_03/Constituicao/Constituiçao.htm

16 Antunes MJM, Egry EY. O Programa Saúde da Família e a reconstrução da atenção básica no SUS: a contribuição da enfermagem brasileira. Rev. Bras. Enferm. 2001 Jan-Mar; 54 (1): 98-107. 